\title{
A Case of Traumatic Brain Injury Presenting with Musical Hallucinations
}

\author{
Yasutaka Kobayashi \\ Department of Rehabilitation Medicine, Fukui General Hospital, Fukui, Japan
}

\section{Keywords}

Musical hallucinations · Traumatic brain injury · Attention disorder

\begin{abstract}
A 67-year-old woman was hospitalized for rehabilitation from the after effects of a traumatic brain injury. In addition to memory impairment, attention disorder, and executive dysfunction, she experienced musical hallucinations. Brain magnetic resonance imaging revealed cerebral contusion in the bilateral temporal lobes and the left frontal lobe. Case reports of musical hallucinations caused by traumatic brain injury are extremely rare.
\end{abstract}

(C) 2017 The Author(s)

Published by S. Karger AG, Basel

\section{Introduction}

Musical hallucinations are the phenomenon in which individuals perceive music in the absence of an external auditory stimulus. Musical hallucinations are rare. In one series of general psychiatric admissions, the prevalence was $0.16 \%$ [1]. In another series of elderly subjects at an audiology clinic, approximately one-third experienced auditory hallucinations, but only 1 subject experienced musical hallucinations [2]. Not only functional psychoses, such as schizophrenia and depression, but also organic brain damage have been pointed out as causes of musical hallucinations. There are few reports of musical hallucinations caused by traumatic brain injury [3]. 


\section{Case Reports in Neurology}

\section{Case Presentation}

A 67-year-old right-handed woman was admitted to our hospital with a chief complaint of musical hallucinations. She had previously been admitted to a different hospital for brain contusion due to a traffic accident. Although she initially had disturbance of consciousness, she recovered gradually. Her consciousness was clear at the time of discharge from the hospital after 7 months, and she had no problems with motor function. However, olfactory dysfunction and right hearing impairment remained, and she was transferred to our hospital for rehabilitation on day 210 after the injury.

At the first visit, there was no abnormality in her motor function and somatosensory function, but olfactory dysfunction and right hearing impairment remained. Right and left mean hearing levels were 55.0 and $30.0 \mathrm{~dB}$ (one-fourth method), respectively. She complained "I forget easily," "I am about to collide with cars on the road," and "I cannot cook well and cannot use the washing machine well." These complaints related to memory impairment, attention impairment, and executive dysfunction, respectively. Her husband said she could not control her emotions. Further, she complained "I always hear music in my brain" and that she was under stress. She often heard nursery rhymes, such as "Furusato" and "Mori no Kumasan," and, at the time of the Olympics, the Japanese national anthem. The music was sometimes mixed with people's voices, and sometimes she heard two kinds of music simultaneously. She did not hear music while sleeping, but she heard it from the time she got up. The volume of the music ranged from high to low, and it was particularly high when she was undergoing dental treatment.

Head magnetic resonance imaging on day 374 after the injury indicated brain contusion in the bilateral temporal lobes and the left frontal lobe (Fig. 1). The electroencephalogram showed no laterality or epileptiform discharges.

Neuropsychological tests were conducted on days 219-247 after the injury. In the Wechsler Adult Intelligence Scale-Third Edition (WAIS-III) [4], the verbal, performance, full IQ, verbal comprehension, perceptual organization, working memory, and processing speed scores were 91, 105, 96, 100,103, 90, and 89, respectively, indicating approximately normal intelligence. In the Wechsler Memory Scale-Revised (WMS-R) [5], the verbal, visual, general memory, attention/concentration, and delayed recall scores were 107, 90, 104, 90, and 89, respectively, indicating approximately normal memory. The standardized profile score on the Rivermead Behavioural Memory Test (RBMT) [6] was 20/24, indicating borderline memory loss. The Clinical Assessment for Attention (CAT) [7] scores were above the cutoff values for all tasks except the memory updating task (4 span), indicating mild disturbance of attention.

Based on the subjective symptoms and the test results, the patient was diagnosed with mild memory impairment, mild attention disorder, and mild executive dysfunction. For rehabilitation, she underwent occupational therapy to stimulate her attention and speechhearing therapy for memory training in ambulatory rehabilitation. She did not want to receive medication for the musical hallucinations. After 2 years and 6 months, the symptoms of memory impairment and executive dysfunction disappeared, but the symptoms of attention disorder remained. Neurological test scores were improved for all tasks except the memory updating task (4 span). She was diagnosed with remaining mild attention disorder. Her musical hallucinations also remained. 


\section{Case Reports in Neurology}

Kobayashi: A Case of Traumatic Brain Injury Presenting with Musical Hallucinations

\section{Discussion}

The patient had mild memory impairment, mild attention disorder, mild executive dysfunction, and musical hallucinations at the first visit. Memory impairment and executive dysfunction were improved after rehabilitation. However, attention disorder and musical hallucinations remained, and she had problems in everyday life.

She often heard nursery rhymes and, at the time of the Olympics, the national anthem. The nursery rhymes were familiar songs from childhood, and the national anthem was being played on television at the time of the Olympics. As previously noted by Golden and Josephs [8], musical hallucinations are related to frequency of sounds throughout the lifetime and to emotionally charged situations. Therefore, a description of music in musical hallucinations may be related to age, experience, environmental factors, etc.

Evers [9] examined 135 previously reported cases of musical hallucinations. The cause was focal brain lesions in 21 cases, which were tumors or blood vessel lesions in all patients except for 2 with infectious lesions. Golden and Josephs [8] identified 393 subjects with musical hallucinations in the Mayo Clinic medical records and divided them into 5 categories based on comorbid conditions that have been associated with musical hallucinations: neurological, psychiatric, structural, drug effect, and not classifiable. They reported 1 case of traumatic brain injury placed in the neurological category. As a mechanism for the onset of musical hallucinations, the release phenomenon is most plausible. In the Charles Bonnet syndrome, visual impairment precipitates the development of visual hallucinations [10]. Similarly, activation of musical memory by auditory blocking is considered one of the causes of musical hallucinations $[11,12]$. Hallucinations may appear if some part of the auditory afferent pathway is impaired. In the present case, the left auditory center was impaired by traumatic brain injury, resulting in the development of musical hallucinations.

In the case of traumatic brain injury due to a traffic accident, disturbance of consciousness is often found in the acute phase, and the doctor's interest is directed only to lifesaving and systemic management. Memory impairment, attention disorder, executive dysfunction, and hallucinations, which are noticed after the recovery of the conscious state, are often considered to be not so important. As a result, there is a high probability that the patient will come to consultation only after problems in daily living have appeared after discharge. When patients with serious brain injuries due to trauma are hospitalized, health care providers need to pay attention to the symptoms seen at an early stage after recovery of consciousness. In the case of traffic accidents, it is also necessary to consider the disability determination services. Monetary compensation is related to the extent to which residual disability affects the patient's daily living in the future. In the case of hallucinations, objective proof of the effect on daily living is difficult to obtain, so it is necessary to link the hallucinations with objective evidence such as brain imaging and hearing test results. In addition, if attention disorder caused by musical hallucinations makes it difficult for the patient to go out alone, as in the present case, daily life may be greatly affected. Health care providers should listen to the patients, estimate the effect that hallucinations will have on their daily lives, and consider treatments and guidance in lifestyle management.

\section{Conclusion}

Musical hallucination is a rare phenomenon, and there are few reports, especially of hallucinations caused by traumatic brain injury. For patients with traumatic brain injury, it is 
necessary to evaluate the neuropsychological dysfunction, as well as to listen to subjective symptoms such as hallucinations, and to use the information for treatment and daily guidance.

\section{Statement of Ethics}

The author has no ethical conflicts to disclose.

\section{Disclosure Statement}

The author has no conflicts of interest to declare. No funding was received for this study.

\section{References}

1 Fukunishi I, Horikawa N, Onai H: Prevalence rate of musical hallucinations in a general hospital setting. Psychosomatics 1998;39:175.

2 Cole MG, Dowson L, Dendukuri N, Belzile E: The prevalence and phenomenology of auditory hallucinations among elderly subjects attending an audiology clinic. Int J Geriatr Psychiatry 2002;17:444-452.

3 Berrios GE: Musical hallucinations. A historical and clinical study. Br J Psychiatry 1990;156:188-194.

4 Fujita K, Maekawa H, Dairoku K, Yamanaka K: The Japanese Version of the Wechsler Adult Intelligence Scale-III. Tokyo, Nihon Bunka Kagakusha, 2007.

$5 \quad$ Sugishita M: The Japanese Version of the Wechsler Memory Scale-Revised. Tokyo, Nihon Bunka Kagakusha, 2007.

6 Watamori T, Hara H, Miyamori T, Eto F: The Japanese Version of the Rivermead Behavioural Memory Test. Tokyo, Chiba Test Center, 2002.

7 Japan Society for Higher Brain Dysfunction: Clinical Assessment for Attention. Tokyo, Shinko Igaku Syuppansya, 2008.

8 Golden EC, Josephs KA: Minds on replay: musical hallucinations and their relationship to neurological disease. Brain 2015;138:3793-3802.

19 Evers S: Musical hallucinations. Curr Psychiatry Rep 2006;8:205-210.

10 Teunisse RJ, Cruysberg JR, Hoefnagels WH, Verbeek AL, Zitman FG: Visual hallucinations in psychologically normal people: Charles Bonnet's syndrome. Lancet 1996;347:794-797.

11 Griffiths TD: Musical hallucinosis in acquired deafness. Phenomenology and brain substrate. Brain 2000;123:2065-2076.

12 Hori H, Terao T, Nakamura J: Charles Bonnet syndrome with auditory hallucinations: a diagnostic dilemma. Psychopathology 2001;34:164-166. 


\section{Case Reports in Neurology}

\begin{tabular}{l|l}
\hline Case Rep Neurol 2018;10:7-11 \\
\hline DOI: 10.1159/000481451 & $\begin{array}{l}\text { C } 2017 \text { The Author(s). Published by S. Karger AG, Basel } \\
\text { www.karger.com/crn }\end{array}$ \\
\hline Kobayashi: A Case of Traumatic Brain Injury Presenting with Musical Hallucinations
\end{tabular}
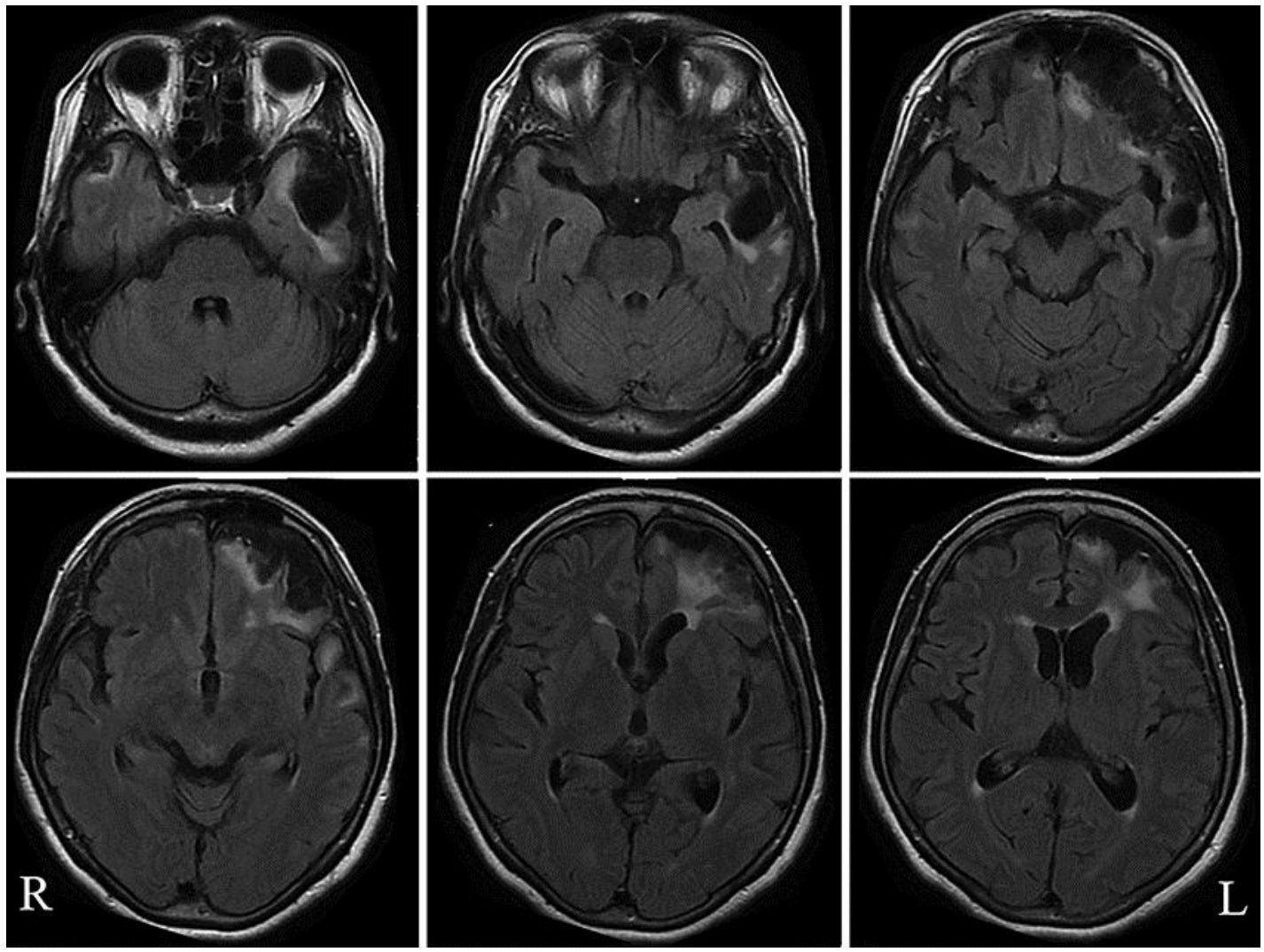

Fig. 1. Head magnetic resonance imaging on day 374 after the injury. Fluid-attenuated inversion recovery imaging reveals cerebral contusion in the bilateral temporal lobes and the left frontal lobe. 